Debate / Controversy

\title{
Elecciones del 26 J: el porqué del no sorpasso
}

\section{J Elections: Reason of no Sorpasso}

\section{José Pablo Ferrándiz}

Metroscopia

jpferrandiz@metroscopia.es

Recibido / Received: 14/12/2016

Aceptado / Accepted: 07/03/2017

\section{RESUMEN}

El artículo sitúa como punto clave para la explicación de la falta de acierto de las encuestas sobre los resultados de las últimas elecciones el denominado sorpasso del PSOE por parte de Podemos. Ya no sólo se trataba de pasar del bipartidismo a un marco de hasta cuatro partidos políticos con la posibilidad de participar en el gobierno, cuestión que demoscópicamente ya había sido asimilada, de manera que las inclinaciones 0 sesgos durante la campaña electoral de cada uno de los cuatro partidos estaban bastante identificados. La tensión estuvo proyectada en si el partido liderado por Pablo Iglesias se convertía en la principal referencia de la izquierda. Una tensión que llevó hasta el último minuto la decisión de muchos votantes.

Palabras clave: Sorpasso, bipartidismo, multipartidismo, Clima Social de Metroscopia.

\section{ABSTRACT}

The article explains the lack of success of the polls on the results of the last elections. The main reason: the so-called sorpaso of the PSOE by Podemos. It was no longer just a question of moving from bipartisanship to a framework of up to four political parties with the possibility of participating in government, an issue that had been democratically assimilated, so that the inclinations or biases during the electoral campaign of each of the four parties were quite identified. The tension was projected in if the party led by Pablo Iglesias became the main reference of the left. A tension that took the decision of many voters to the last minute.

Key words: Sorpasso, bipartisanship, multiparty, Metroscopia Social Climate.

*Autor para correspondencia / Corresponding author: José Pablo Ferrándiz. Metroscopia. Calle General Yagüe, 6 bis, 28020 Madrid, España/Spain. Sugerencia de cita / Suggested citation: Ferrándiz, J. P. (2017). Elecciones del 26 J: el porqué del no sorpasso. Revista Española de Sociología, 26 (3 Supl.), 129-137. 


\section{INTRODUCCIÓN}

El 26 de junio de 2016 se celebraron en España las decimoterceras elecciones generales desde la restauración de la democracia en nuestro país. Los resultados de estos comicios confirmaron el cambio del sistema de partidos español que se había producido tan solo seis meses antes, en las elecciones del 20 de diciembre de 2015, cuando se pasó de un bipartidismo imperfecto ${ }^{1}$ vigente en las últimas décadas y caracterizado por la alternancia en el Gobierno de uno de los dos principales partidos (PP y PSOE), a un cuatripartidismo competitivo con cuatro fuerzas políticas - PP, PSOE, Podemos y Ciudadanos - orientadas hacia el gobierno, es decir, con disponibilidad para promover y construir coaliciones gubernamentales viables (esto es, políticamente razonables y numéricamente posibles). La falta de acuerdo entre los partidos políticos para investir a un presidente del Gobierno provocó la repetición de las elecciones seis meses después. La única e importante novedad entre una elección y otra fue el acuerdo entre Unidad Popular y Podemos para concurrir juntos en una única candidatura denominada Unidos Podemos. Su aspiración era, tal y como, por otro lado, estimaban la amplia mayoría de sondeos preelectorales publicados, conseguir superar al PSOE en número de votos y/o de escaños. Un hecho que finalmente no llegó a producirse como consecuencia, y ésta es la hipótesis que aquí se plantea, de la combinación de varios factores.

\section{ANTECEDENTES: LAS ELECCIONES GENERALES DEL 20 DE DICIEMBRE DE 2015}

Las elecciones generales celebradas en España el 20 de diciembre de 2015 —las duodécimas desde la restauración de la democracia - arrojaron unos resultados inéditos hasta ese momento en la historia electoral de nuestro país. Por pri-

1 La expresión "imperfecto" fue acuñada por Giorgio Galli (1966) para definir el sistema político italiano de la década de los sesenta. mera vez desde las elecciones constituyentes de 1977 cuatro partidos políticos lograban superar el $10 \%$ de los votos válidos emitidos sin que ninguno de ellos consiguiera alcanzar el $30 \%$. El PP obtuvo el $28.7 \%$, el PSOE el $22 \%$, Podemos el $20.7 \%$ y Ciudadanos el $13.9 \%$. Además, nunca antes la diferencia entre el primer y el cuarto partido, tanto en número de votos como en número de escaños, había sido tan estrecha: 15.8 puntos porcentuales y 83 diputados. Asimismo, el número de escaños logrado por la formación política que consiguió más votos (en este caso, el Partido Popular) fue el más bajo de todos los procesos electorales anteriores (123). Y fue, también, la primera vez que el segundo partido se quedaba por debajo de los 100 diputados (el PSOE logró 90). Por otro lado, nunca antes la suma de porcentajes de votos y la suma de escaños de los dos partidos principales habían sido tan reducidas: $50.7 \%$ y 213 diputados (un $60.9 \%$ del total de los 350 escaños que componen el Congreso de los Diputados). Y otra novedad: fue la primera vez que dos partidos nuevos, debutantes en unas elecciones generales, obtenían tantos votos y escaños como los logrados por Podemos y por Ciudadanos: entre los dos sumaron casi nueve millones de votos (5 189333 votos los primeros y 3500446 los segundos) y casi un tercio de los diputados del Congreso (109: 69 y 40, respectivamente). También fue la primera vez que los españoles se fueron a la cama la noche electoral sin saber quién iba a ser su próximo presidente del Gobierno². De hecho, por primera vez en la historia de España, hubo que repetir las elecciones generales por la falta de acuerdo entre los diferentes partidos políticos con representación parlamentaria para elegir a un

2 En las elecciones generales de 1996, las primeras ganadas por el PP, se tardaron dos meses desde su celebración en investir presidente a José María Aznar (las elecciones se celebraron el 3 de marzo y la investidura se produjo el 4 de mayo). En todo caso, en aquella ocasión existían pocas dudas de que el candidato popular fuera a ser investido presidente. Solo parecían existir dudas acerca de dónde provendrían sus apoyos. Finalmente, Aznar consiguió el voto a favor de, además de sus 156 diputados, los 16 de CiU, los 5 del PNV y los 4 de CC. 
presidente. Tras las elecciones de 2015, el 13 de enero de 2016 se iniciaba la XI Legislatura que finalizó el 3 de mayo de este mismo año pasando a ser — otra novedad — la más corta de la democracia española: solo duró 111 días.

La participación electoral fue del 73.0 \% sobre el total del censo de residentes en España, porcentaje que descendió hasta un $69.6 \%$ una vez contabilizados los votos de los españoles residentes en el extranjero. La participación fue, así, inferior a la media de los 12 procesos electorales celebrados hasta ese momento en España desde la restauración de la democracia (que es de $74.2 \%$ ) y más baja de lo estimado por la mayoría de sondeos lo que explica, en parte, algunas diferencias entre los sondeos preelectorales y los resultados finales ${ }^{3}$.

Los resultados de las elecciones del 20 de diciembre de 2015 permiten afirmar, siguiendo la tesis de Morgan (2011), que el sistema de partidos español colapsó. En su libro Bankrupt of representation and party system collapse, la autora mantiene la teoría de que para que un sistema de partidos ${ }^{4}$ colapse tienen que producirse de manera simultánea y en un corto período de tiempo dos circunstancias: el desmoronamiento partidista y la transformación del sistema. Estos dos acontecimientos se han producido en el caso español en apenas cuatro años: los que abarca la $X$ Legislatura (transcurrida desde el 13 de diciembre de 2011 al 27 de octubre de 2015).

3 La participación electoral es decisiva en los resultados electorales por una sencilla razón: la abstención es selectiva, esto es, no se reparte, obviamente, de manera proporcional entre todos los electorados. Hay unos partidos que cuentan con un electorado más fiel (al partido y a las urnas) y otros, por el contrario, tienen votantes más volátiles y electoralmente más hipotensos. Por eso, el dato de la participación ya ofrece algunas pistas por sí solo de a qué partidos les puede ir mejor y a cuáles peor en unas elecciones. La diferencia en votos absolutos entre la participación estimada y la que hubo finalmente fue de alrededor de un millón de electores.

4 Se utiliza la definición de sistema de partidos de Sartori (1980): "el sistema de interacciones que es resultado de la competencia entre partidos".

\section{EVOLUCIÓN DE LOS SONDEOS DE OPINIÓN DESDE EL 20D HASTA EL 26J}

La XI Legislatura que se inició el 13 de enero de 2016 llegaba a su fin tan solo 111 días después, el 3 de mayo, convirtiéndose en la de menor duración desde la restauración de la democracia en nuestro país. Tres semanas después del 20D, los datos que arrojaba el Clima Social de enero de 2016 de Metroscopia permitían afirmar, sin lugar a dudas, que los ciudadanos que habían propiciado el cambio del sistema de partidos en las elecciones de diciembre de 2015 se reafirmaban en su decisión. Un 61 \% de los españoles se declaraba satisfecho con el resultado de las elecciones: pedían el fin del bipartidismo y eso es lo que se había producido. En esos días transcurridos desde los comicios, el español medio distaba mucho de experimentar la sensación de que el mayor pluralismo parlamentario que se había producido equivaliera necesariamente a ingobernabilidad. Prueba de ello era que cuando se les planteaba la posibilidad de una repetición de las elecciones el resultado que los datos permitían estimar como más probable reforzaba, en vez de debilitar, al cuatripartidismo emergido de las urnas.

Los líderes de las principales fuerzas políticas fueron incapaces de ponerse de acuerdo para investir un presidente y conformar un Gobierno en el plazo establecido por la Constitución. Tras consultar a todos los líderes políticos con representación parlamentaria y la negativa de Rajoy a presentarse a una investidura por no contar con los apoyos suficientes, el 2 de febrero el Rey Felipe IV propuso como candidato al líder del PSOE, Pedro Sánchez. El 24 de febrero los socialistas firmaron un acuerdo con Ciudadanos para la investidura de Sánchez como presidente, pero entre los dos partidos no lograban sumar la mayoría suficiente para lograrlo: el PSOE con 90 diputados y Ciudadanos con 40 (130) necesitaban, así, el apoyo o la abstención de otras formaciones políticas (básicamente Podemos). Pero en la primera votación, en la que el candidato necesita mayoría absoluta de votos a favor, Sánchez solo logró el voto afirmativo de sus diputados y los de Ciudadanos (130), 


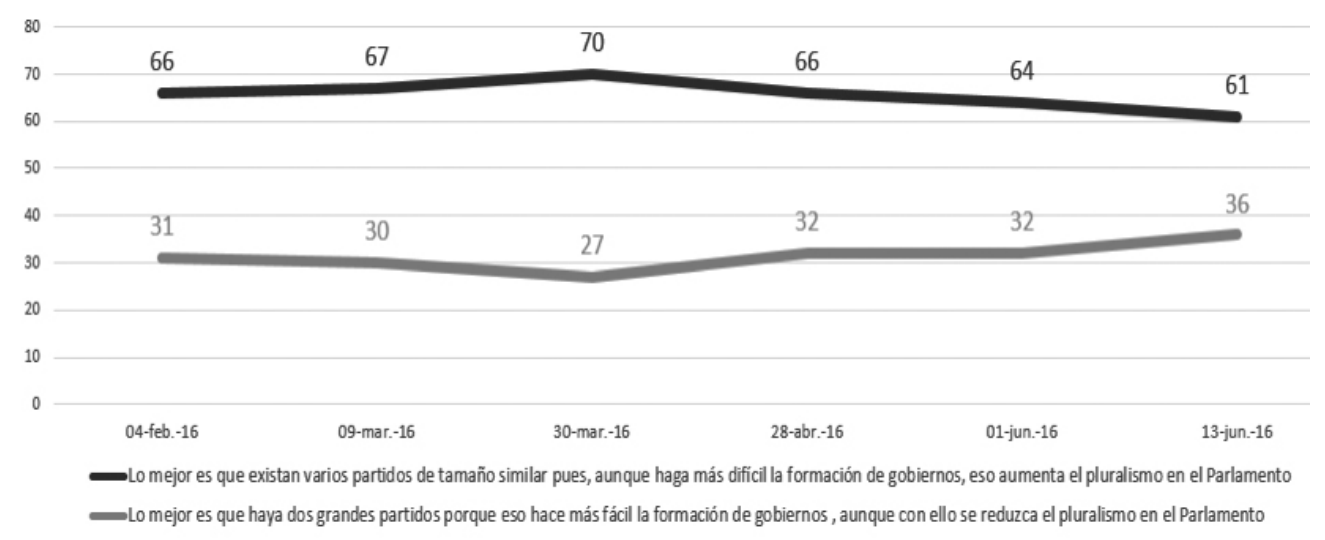

Figura 1. Evolución de las pertenencias de la ciudadanía española por un modelo bipartidista y por un modelo multipartidista en el Parlamento

Fuente: Banco de Datos de Metroscopia

recibió 219 votos en contra y una abstención (correspondiente a la diputada de Coalición Canaria). En la segunda votación — que tuvo lugar dos días después: el 4 de marzo- el candidato no logró la mayoría simple de votos a favor necesaria para ser investido en segunda votación. Sánchez obtuvo 131 síes (los 90 de los diputados del PSOE, los 40 de los de Ciudadanos y el de la diputada de CC). El resto de fuerzas políticas parlamentarias votaron en contra (219). Fue la primera vez en la historia democrática de España que un candidato a la presidencia del Gobierno no lograba la confianza del Congreso de los Diputados en ninguna de las dos votaciones para ser investido presidente. Como en los dos meses posteriores ni Sánchez ni ningún otro candidato consiguieron reunir los apoyos necesarios para ser propuestos por Felipe IV para una nueva sesión de investidura, se convocaron automáticamente nuevas elecciones generales tal y como establece la Constitución española.

Finalmente, el 26 de junio se repitieron las elecciones a las que concurrieron como candidatos de las cuatro principales fuerzas políticas, las protagonistas del cuatripartidismo, los mismos líderes que en 2015: Rajoy por el PP, Sánchez por el PSOE, Iglesias por Podemos y Rivera por Ciuda- danos. La gran novedad de esas elecciones fue la unión de Podemos, Izquierda Unida y Equo en una coalición que se presentó bajo la denominación de Unidos Podemos.

A pesar de la falta de acuerdo y de haber estado seis meses sin Gobierno, la amplia mayoría de los ciudadanos seguía pensando - en la pregunta que se realizaba de manera mensual el porcentaje nunca bajó del $60 \%$ - que lo mejor es que en el Parlamento español siguieran existiendo varios partidos de tamaño no muy diferente pues eso aumenta el pluripartidismo aunque implicara una mayor dificultad para formar gobiernos. Menos de un tercio de los españoles creía que lo mejor es que hubiera dos grandes partidos porque aunque con ello se redujera el pluralismo en el Parlamento, hacía más fácil la formación de gobiernos (véase Figura 1). De esta cultura bipartidista era partidaria la mayoría solo de los votantes del PP. Los votantes de Podemos, del PSOE y de Ciudadanos eran mayoritariamente partidarios de la opción multipartidista 5 .

5 A este respecto puede leerse el análisis de Francisco Camas García "Tres culturas políticas en España" (http:// metroscopia.org/tres-culturas-politicas-en-espana/). 

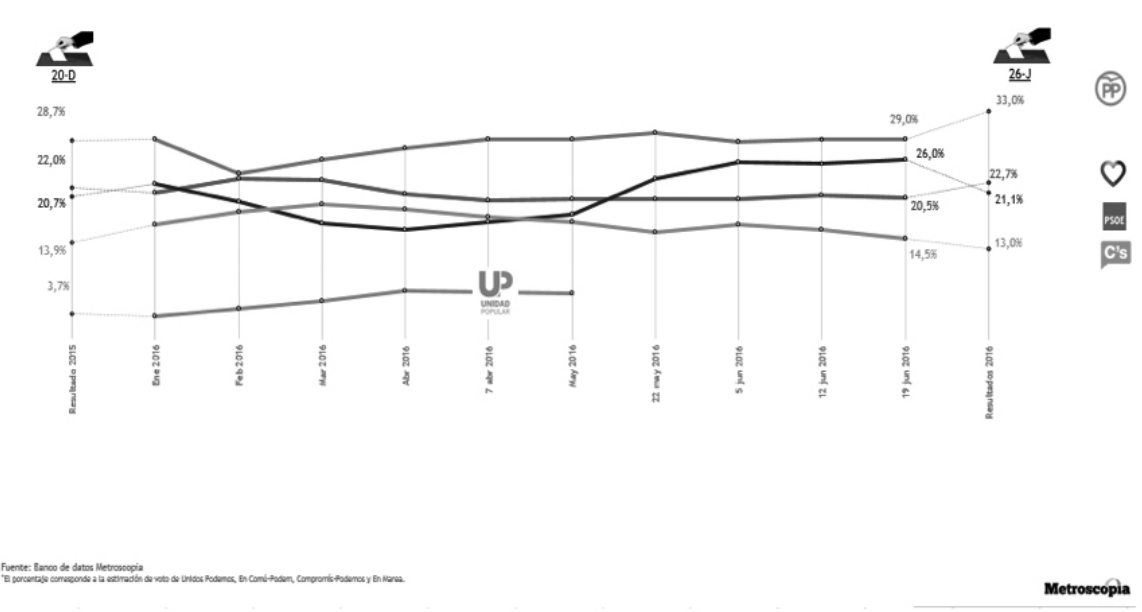

Figura 2. Evolución de la estimación del resultado electoral y resultados electorales del 20D y 26J (en porcentajes sobre voto válido)

Las diez oleadas del Barómetro de Clima Social (véase Figura 2) que se llevaron a cabo desde enero a junio de 2016 (cuando se publicó el último preelectoral de Metroscopia en EL PAÍS) ponen de manifiesto la configuración político-actitudinal de la sociedad española el ínterin entre ambos comicios y cuál fue la traslación de estos estados de opinión al comportamiento electoral en las elecciones del 26J. Bajo esta intención descriptiva subyace la hipótesis metodológica de que las encuestas de opinión pública (las realizadas sobre temas de la agenda de asuntos públicos) reflejan razonablemente bien la dinámica política y de que ayudan tanto a estimar como a explicar los resultados electorales ${ }^{6}$.

En el caso del PP, su estimación de voto para el caso de una repetición electoral no se distanció mucho a lo largo de las 10 oleadas de su resultado real de 2015. A excepción de las oleadas de febrero, marzo y abril, en las que su porcentaje de voto estimado se situó por debajo del 28.7 \% logrado el 20D (un hecho que fue consecuencia, sobre todo, del primer plano informativo que tuvo durante los

6 Algo que puso de relieve Rafael López Pintor (1982) en su ya clásico estudio de la opinión pública que abarca desde finales del franquismo hasta los primeros años de la democracia. primeros meses del año el Caso Taula —un caso de corrupción política vinculado al PP valenciano-), las perspectivas demoscópicas del PP de cara al 26J siempre fueron mejores que las del 20D.

En el caso del PSOE su trayectoria demoscópica indicaba lo contrario. A excepción de las oleadas del mes de febrero y marzo — coincidiendo con el mayor protagonismo de su líder, Pedro Sánchez, por su decisión de presentarse a una votación de investidura - el resto de las oleadas el PSOE obtenía resultados estimados siempre por debajo de su resultado real de 2015.

Ciudadanos, por su parte, parecía repetir algo que parece haberse convertido en algo habitual en su, hasta ahora, corta carrera política en el ámbito nacional: los apoyos electorales que obtiene en las encuestas se van reduciendo conforme se acerca el momento de la elección. Esta ocasión no supuso una excepción: del $19.5 \%$ estimado en la oleada de marzo de 2016 (donde se vio favorecido por su apoyo a la investidura de Sánchez) pasó al 14.5 \% en el preelectoral publicado una semana antes de los comicios.

El caso que, probablemente, merece ser resaltado en mayor medida es el de Podemos y Unidad Popular, las dos formaciones políticas que finalmente llegaron a un acuerdo para presentarse bajo unas mismas siglas: Unidos Podemos. Al contrario de lo 
que le ocurre a Ciudadanos, la formación liderada por Pablo Iglesias sufre demoscópicamente en períodos fuera de campaña electoral. En las oleadas de febrero a mayo del Clima Social obtuvo porcentajes de voto estimados por debajo de su resultado del 20D al tiempo que Unidad Popular lograba, en esos mismos meses, un crecimiento lento pero continuo.

No obstante, desde el momento en el que se conformó Unidos Podemos, la estimación de voto a la nueva coalición fue creciendo hasta situarse en el sondeo preelectoral del 19 de junio en un $26.0 \%$. Este dato suponía que en las elecciones de 2016 las marcas Podemos y Unidad Popular sumaban más juntas que lo que por separado había logrado cada una en las elecciones de 2015 .

\section{LAS ELECCIONES GENERALES DEL 26J}

Aunque con algunos matices, los resultados de las elecciones del 26J mantuvieron el sistema cuatripartidista ${ }^{8}$ emanado de las urnas en los comicios del 20 de diciembre de 2015. De nuevo los mismos cuatro partidos que seis meses antes obtuvieron porcentajes sobre voto válido por encima del $10 \%$ pero, esta vez, uno de ellos superó el $30 \%$. El PP volvió a ser el partido más votado (el ganador aritmético) y con el $33.0 \%$ de los votos (4.3 puntos más que el 20D) fue el único de los cuatro que mejoró sus resultados con respecto a los obtenidos seis meses antes. A pesar de que la participación electoral descendió 3.4 puntos, los populares consiguieron casi setecientos mil votos más que le permitieron añadir 14 diputados a los 123 logrados en 2015 (en total, 137). El PSOE volvía a ser la segunda fuerza política pero perdía más de cien mil votos y cinco diputados con respecto a los anteriores comicios lo que supo-

7 Hay que tener en cuenta que si se sumaban el número de votos y de escaños de Unidad Popular y Podemos en las elecciones del 20D de 2015 el resultado era superior al logrado por el PSOE. En este sentido, algunos análisis concluían que el sorpasso ya se había producido en esos comicios y que el resultado del 26J iba a ser la confirmación de ese hecho.

8 Respecto a las transferencias de voto que se produjeron en las elecciones del 26J puede leerse el análisis "Fidelidades y Fugas para explicar el 26J" (http://metroscopia.org/ fidelidades-y-fugas-para-explicar-los-resultados-del-26j/). nía, otra vez, su peor resultado histórico en unas elecciones generales. En todo caso, como ya se ha comentado anteriormente, la política tiene mucho de gestión de expectativas, y como los socialistas no fueron superados ni en número de votos ni en escaños por Unidos Podemos, en contra de lo que todas las encuestas preelectorales estimaban como más probable, su mal resultado quedó momentáneamente enmascarado. En efecto, la coalición de Podemos con Izquierda Unida y Equo, no solo no sumó los votos que cada partido había conseguido por separado en las elecciones de 2015 (de ser así, el sorpasso al PSOE se hubiera producido) sino que restó. En número de votos pasaron de superar los seis millones el 20D a quedarse en prácticamente cinco. Eso sí, consiguieron mantener el mismo número de diputados que habían obtenido por separado: 71. Ciudadanos, por su parte, perdió en torno a cuatrocientos mil votos y ocho diputados.

Con respecto al último preelectoral publicado por Metroscopia ${ }^{9}$ el 19 de junio el PP logró cuatro puntos más de su resultado estimado, el PSOE 2.2 puntos más, Unidos Podemos 4.9 puntos menos y Ciudadanos 1.5 puntos menos. Pero, sin duda, Io más destacado fue que no se produjo el sorpasso de la formación política liderada por Iglesias a los socialistas que los sondeos estimaban. Varios, parecen ser, los motivos.

\section{¿POR QUÉ NO SE PRODUJO EL SORPASSO?}

En primer lugar, cabe incidir en el hecho de que los sondeos preelectorales no tienen la función de adivinar un resultado electoral por una sencilla razón: no pueden hacerlo. Quien crea que los sondeos "fallan", porque no son capaces de acertar el resultado electoral es que desconoce las cualidades y las limitaciones de esta herramienta. Los sondeos preelectorales estiman tendencias electorales y, en todo caso, muestran el estado de la opinión pública en un momento concreto. Pero es obvio que la opinión pública cambia. Y hay que tener en cuenta, además,

9 Todos los sondeos de Metroscopia mencionados en este trabajo pueden consultarse en su página web: www. metroscopia.org 
que en España, desde que se ha ampliado la oferta partidista con la entrada en la escena política de dos nuevas fuerzas políticas — primero Podemos $\mathrm{y}$, posteriormente, Ciudadanos - ha aumentado con respecto a procesos electorales anteriores tanto la volatilidad electoral como la incertidumbre y la duda. Y esto ha propiciado que cada vez sean más los electores que retrasan su decisión de voto acercándola cada vez más al día de las elecciones ${ }^{10}$.

En este sentido, cabe señalar que pocos días antes de las elecciones del 26J, siete de cada diez españoles convocados a las elecciones decían que con total seguridad iban a acudir a votar. Pero de ellos, había un porcentaje que todavía no se había decantado por una opción política concreta. Son los denominados decididos indecisos. En ese momento representaban un $13 \%$, la mitad que en las elecciones del 20D y el porcentaje más bajo de las últimas tres elecciones generales: en 2008 eran el $19 \%$, en las de 2011 el $21 \%$ y en las de 2015 el $25 \%$. Este era, sin duda, un dato relevante: porque ponía de manifiesto que el voto de los españoles estaba en esos momentos mucho más asentado de lo que lo estaba apenas seis meses antes. Los que dudaban de cara al 26J eran muchos menos y, por tanto, su comportamiento parecía que iba a ser algo menos decisivo en el resultado final de lo que pudo serlo en 2015. Con todo, se trataba de más de tres millones de españoles que estaban siendo el centro de atención de todas las formaciones políticas en esos últimos días de campaña. Para unos partidos, lograr su apoyo significaría mejorar sus resultados. Para otros, evitar el desastre. Un análisis del perfil de estos decididos indecisos arrojaba algunas claves que ayudaban a entrever cuál podría llegar a ser finalmente su comportamiento electoral más probable. El grupo más numeroso de decididos indecisos (23\%) dudaba entre votar al PSOE 0 a Unidos Podemos; un $15 \%$ no sabía todavía si apoyar al PP 0 a Ciudadanos; un $6 \%$ estaba indeciso entre el PP y el PSOE y otro $6 \%$ duda entre el PSOE y Ciudadanos. Los socialistas eran, así, el partido que estaba presente en un mayor número de opciones.

10 Un claro hándicap para los sondeos preelectorales pues en España está prohibida su publicación los cinco días previos al día de los comicios.
Por el perfil descrito no parecía descabellado pensar que una gran parte de los decididos indecisos, de acabar votando, lo hiciera en favor del PSOE Io que, sin duda, mejoraría el resultado estimado por Metroscopia en el último barómetro preelectoral ${ }^{11}$. Ahora bien, ¿tanto como para evitar el sorpasso? Parece ser que así fue. En un sondeo postelectoral llevado a cabo por Metroscopia ${ }^{12}$ tras los comicios arroja algunas claves de porqué, finalmente, Podemos no se alzó con la segunda posición.

Un primer dato tiene que ver con que una parte de los electores de entre 18 y 54 años de edad que manifestaba en los sondeos previos su clara intención de acudir a votar a la coalición Unidos Podemos, finalmente no lo hizo: 0 bien optó por otra opción política 0 bien se abstuvo. Todo parece indicar que Unidos Podemos no fue capaz de atraer a tantos jóvenes como mostraban las encuestas preelectorales, aunque, en todo caso, siguió concentrando buena parte de sus votantes en este grupo de edad. No obstante, lo más destacado es la pérdida de electores de mediana edad que sufrió: la diferencia entre quienes mostraban inclinación por la coalición antes de las elecciones (21.5\%) y quienes dicen que finalmente le dieron su voto (13.5\%) es de -8 puntos porcentuales. En el caso de los jóvenes, el diferencial es de -5.5 . Estas fugas se produjeron, precisamente, en

11 El sondeo preelectoral puede consultarse en http://metroscopia.org/mas-que-ganar-es-ser-necesario/

12 El sondeo se ha efectuado mediante entrevistas telefónicas a una muestra nacional de personas residentes en España, mayores de 18 años y con derecho a votar en elecciones generales. Se han completado 4000 entrevistas a través de llamadas a teléfonos móviles seleccionados de forma aleatoria a partir de un generador automático de números telefónicos. Posteriormente se han calibrado los datos a partir de una ponderación múltiple por las variables sexo, edad, hábitat y región (comunidad autónoma). La eficiencia de la ponderación es del $73.7 \%$, de modo que la muestra efectiva equivale a 2948 entrevistas. El error de muestreo, para un nivel de confianza del $95.5 \%$ (que es el habitualmente adoptado) y asumiendo los principios del muestreo aleatorio simple, en la hipótesis más desfavorable de máxima indeterminación ( $p=q=50 \%$ ), es de \pm 1.6 puntos (tras la ponderación es de \pm 1.8 puntos). La recogida de información y el tratamiento de la misma han sido llevados a cabo íntegramente en Metroscopia. La fecha de realización del trabajo de campo es del 29 de junio al 4 de julio de 2016. 


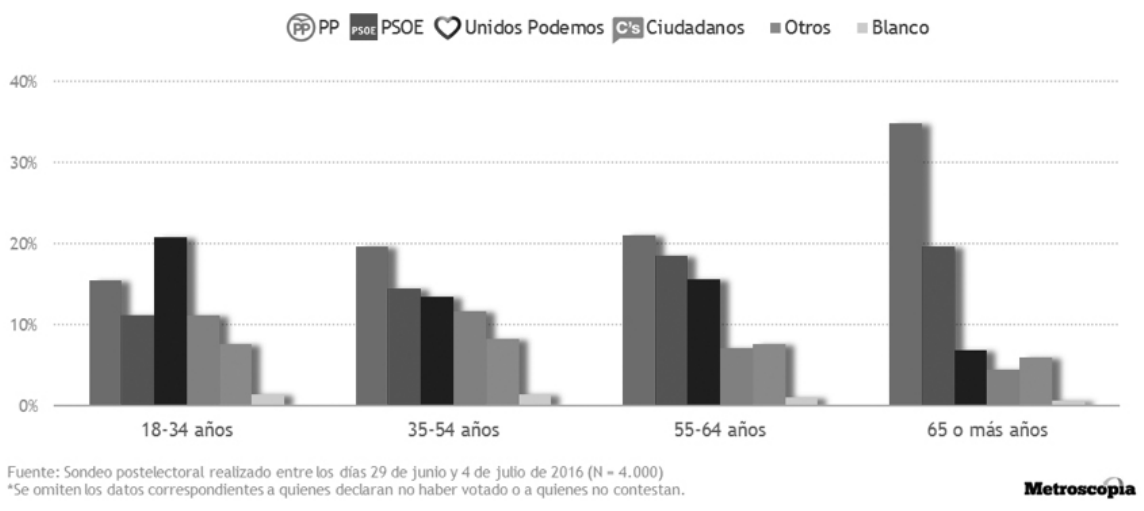

Figura 3. Recuerdo de voto. del 26J según la edad

los segmentos en los que la abstención declarada se incrementó más (entre 9 y 10 puntos en el intervalo de 18 a 54 años, y entre 3 y 6 puntos en el de 55 años 0 más, véanse Figuras 3, 4 y 5). En este sentido, parece haber una clara relación entre quienes se abstuvieron y quienes en el último momento decidieron no votar al partido de Iglesias. Una abstención atribuible principalmente a anteriores votantes de Unidad Popular que finalmente no votaron a la nueva coalición ${ }^{13}$.

Un segundo dato tiene que ver con el electorado del PSOE. Los socialistas no consiguieron retener a una pequeña parte de electores mayores (más de 54 años), pero compensaron relativamente esas pérdidas con un mejor resultado entre la gente joven y la de mediana edad. Además, el PSOE logró un aporte final de votos procedente de su tradicional electorado que si bien en las encuestas decía que no iba a votar a los socialistas, acabó haciéndolo motivado por la posibilidad del sorpasso que estimaban las encuestas: en el postelectoral de Metroscopia,

13 Un mes antes de las elecciones del 26J, los sondeos arrojaban que en torno a un tercio de los votantes de Unidad Popular no tenía intención de votar a la nueva coalición. Según se acercaba la cita electoral este porcentaje se fue reduciendo lo que, en gran medida, motivó la sobreestimación electoral a Unidos Podemos. El postelectoral de Metroscopia arrojó, sin embargo, que en torno a un tercio de los votantes de Unidad Popular no acabó votando la lista de Unidos Podemos. un $20 \%$ de votantes del PSOE dice que este hecho le influyó en su decisión final de voto.

$Y$ un tercer elemento tiene que ver con el Brexit. Solo tres días antes de las elecciones en España, se celebró un referéndum en el Reino Unido sobre su permanencia en la Unión Europea. La victoria de los partidarios de la salida de la UE llenó los medios de comunicación de informaciones marcadamente negativas sobre las consecuencias económicas de esta decisión. Un hecho que, sin duda, introdujo en plena campaña electoral, a tan solo 48 horas de las elecciones, un clima de incertidumbre que benefició a los partidos tradicionales y perjudicó a los emergentes, en especial, a Unidos Podemos: el postelectoral de Metroscopia apunta a que para un $16 \%$ de Ios votantes del PP y un $14 \%$ de los del PSOE las informaciones sobre el Brexit le habían influido en su decisión final de voto (frente a solo un $6 \%$ en el caso de los votantes de UP). En este sentido cabe señalar que el aumento del PP con respecto a lo estimado en el último sondeo de Metroscopia tuvo que ver con la capacidad de los populares de atraer a última hora a electores jóvenes (menores de 35 años) que pocos días antes no estaban dispuestos a acudir a votar 0 que, si tenían pensado hacerlo, preferían la opción de Ciudadanos. Todo apunta a que tanto la abstención (que fue del 30.2 \%) como las transferencias de voto que finalmente se produjeron acabaron afectando, así, más a los partidos emergentes — Unidos Podemos y Ciudadanosque a los partidos tradicionales - PP y PSOE-. 


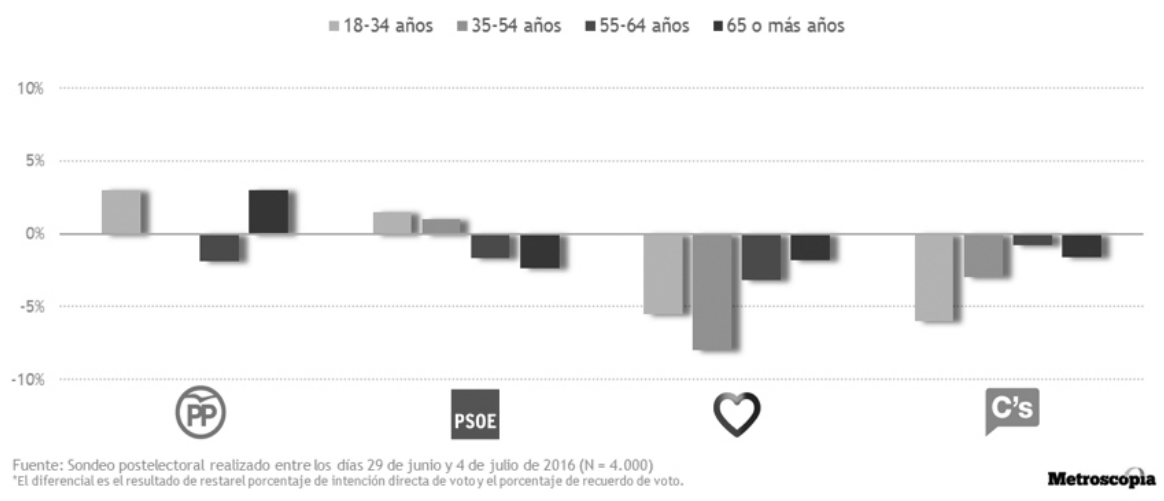

Figura 4. Diferencias entre quienes tenían intención de votar a PP, PSOE, Podemos y Ciudadanos y finalmente dicen que lo hicieron (en puntos porcentuales)

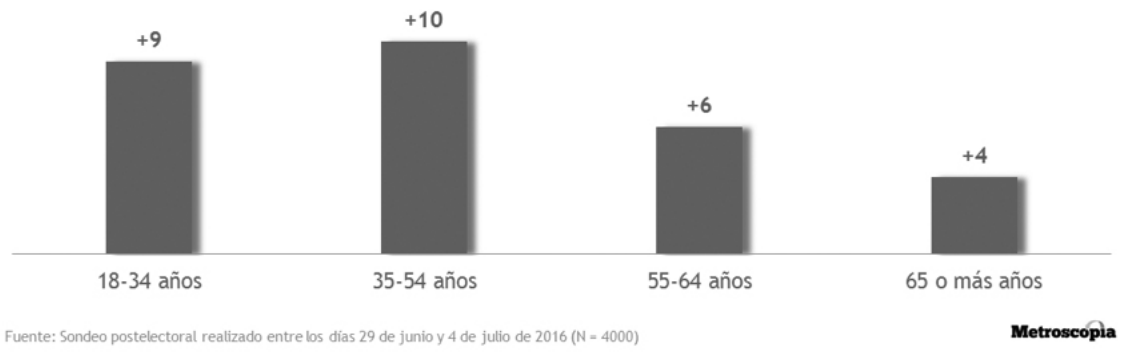

Figura 5. Diferencias entre quienes tenían intención de abstenerse el 26J y finalmente dicen que se abstuvieron (en puntos porcentuales)

\section{REFERENCIAS BIBLIOGRÁFICAS}

Galli, G. (1966). I/ bipartidismo imperfecto: comunista e democristiani, II Mulino: Bolonia.

Linz, J. J., Montero, J. R. (2013). Los sistemas de partidos en España en el último cuarto del Siglo XX, en J. R. Montero y T. Jeffrey Miley (eds.), Obras escogidas 6. Partidos y élites políticas en España (pp. 627-733). Centro de Estudios Políticos y Constitucionales: Madrid.

López Pintor, R. (1982). La opinión pública española: del franquismo a la democracia. Centro de Investigaciones Sociológicas: Madrid.
Morgan, J. (2011). Bankrupt Representation and Party System Collapse. Pennsylvania: The Pennsylvania State University Press.

Sartori, G. (1980). Partidos y sistema de partidos 1. Madrid: Alianza Editorial.

Seawright, J. (2012). Party-system collapse. The roots of crisis in Peru and Venezuela. Stanford, California: Stanford University Press,

Urquizu, I. (2016). La crisis de representación en España. Madrid: Editorial Catarata. 
